\title{
ON THE SEPARATION OF THE PLANE BY IRREDUCIBLE CONTINUA $\dagger$
}

\author{
BY W. A. WILSON
}

1. Introduction. This question was investigated by $\mathrm{A}$. Rosenthalf in 1919. His principal results may be stated as follows. If $F$ is the union of two bounded continua, $C_{1}$ and $C_{2}$, which are irreducible between the points $a$ and $b$ and have no other common points, then the complement of $F$ with respect to its plane consists of two principal component regions (Hauptgebiete), each of which has $F$ as its frontier, and possibly of a number of secondary component regions (Nebengebiete), each of which has its frontier wholly in either $C_{1}$ or $C_{2}$.

It is a simple matter to construct two irreducible continua which together constitute the frontier of precisely two of the complementary regions, but which have more than two points in common. For example, let $b m c$ and $b n c$ be two complementary arcs of a circumference, and let $a m^{*}$ and $a n^{*}$ be two wavy lines intersecting only at $a$ and approaching asymptotically $b m c$ and $b n c$, respectively. Then $C_{1}=a m^{*}+b m c$ and $C_{2}=a n^{*}+b n c$ are both irreducible between $a$ and $b$ and divide the plane into three regions, of which two only have $C_{1}+C_{2}$ as their frontier. But $C_{1}$ and $C_{2}$ have in common three points, not two.

In this particular case, to be sure, Rosenthal's theorem can be used, for $C_{1}+C_{2}$ can be expressed as the union of two continua, irreducible between a pair of points and intersecting only in these points. This is possible here because $C_{1}$ and $C_{2}$ can each be separated in to two irreducible continua having only one common point. Such a decomposability,

$\dagger$ Presented to the Society, October 30, 1926.

$\ddagger$ (A) A. Rosenthal, Teilung der Ebene durch irreduzibele Kontinua, Sitzungsberichte der Münchener Akademie, 1919, pp. 91-109. 
however, is not a general property of irreducible continua, even when no indecomposable continua are involved. Therefore a removal of the requirement that the two irreducible continua have but two points in common is a real extension of the theorem quoted above.

It is the purpose of the present article to give some general conditions under which this extension is possible and also to investigate the frontiers of the secondary regions. The principal results will be found in $\$ \S 5$ and 8 .

In certain parts of the work free use is made of the oscillatory set of a continuum about a point and its properties, which have been developed by the author in the papers listed below.* The notation of these papers is also used.

2. Some Generalizations. For what follows it is convenient to extend the notion of a continuum irreducible between two points in two ways which must be kept distinct.

Definition I. If $A$ is a closed sub-set of the continuum $C$, then $C$ is irreducible about $A$ if no proper sub-continuum of $C$ contains $A$.

DEFINITION II. If $\alpha$ and $\beta$ are closed sets without common points and the continuum $C$ contains one or more points of both sets, then $C$ is irreducible between $\alpha$ and $\beta$ if no proper sub-continuum of $C$ contains points of both sets.

The second definition is due to Miss Anna M. Mullikin. $\dagger$ It is readily seen that if $A$ or $\alpha+\beta$ consists of two points, both definitions yield the ordinary continuum irreducible between two points. The following properties will be used.

LEMMA I. If $C$ is a bounded continuum irreducible about the sum of the two continua $A$ and $B$, which have no common points, then $C-(A+B)$ is connected.

* (B) On the oscillation of a continuum, Transactions of this Society, vol. 27.

(C) Some properties of the irreducible continuum, ibid., vol. 28.

(D) On the structure of the irreducible continuum, American Journal, vol. 48 .

$\dagger(\mathrm{E})$ Anna M. Mullikin, Certain theorems relating to plane connected sets, Transactions of this Society, vol. 24. 
The proof given by Rosenthal (loc. cit., p. 104) for the case where $A$ and $B$ are points is immediately applicable.

LEMMA II. Let $C$ be bounded and closed and contain points of both the closed sets $\alpha$ and $\beta$. Let $\alpha \cdot \beta=0$. Then $C$ can be separated into two closed sets $C_{1}$ and $C_{2}$, such that $C_{1} \cdot C_{2}$ $=\alpha \cdot C_{2}=\beta \cdot C_{1}=0$, or else $C$ contains a sub-continuum irreducible between $\alpha$ and $\beta$.

This theorem is stated and proved in an equivalent form by Miss Mullikin (loc. cit., p. 147).

Lemma III. Let $C$ be a bounded continuum irreducible between the closed subsets $\alpha$ and $\beta$, which have no common points. Let $A$ be the oscillatory set of $C$ about some point a of $\alpha$. Then $A$ contains $\alpha$ and, if $D$ is a sub-continuum of $C$ containing a point $x$ of $C-A$ and a point $a^{\prime}$ of $\alpha, D$ contains $A$.

Proof. We omit the trivial case that $C$ is indecomposable. If $A$ is complete, $C-A$ is a semi-continuum by a theorem proved elsewhere.* Obviously it contains $\beta$ and hence can contain no point of $\alpha$. Let $E$ be a sub-continuum of $C-A$ joining $x$ and $\beta$. Then $D+E$ joins $\alpha$ and $\beta$, and hence equals $C$. As $A \cdot E=0, D$ contains $A$.

If $A$ is not complete, it is indecomposable and is not a continuum of condensation. $\dagger$ Then $\overline{C-A}$ is a proper subcontinuum $\ddagger$ of $C$ and contains $\beta$. Hence it contains no point of $\alpha$. Thus $A$ contains $\alpha$ in this case too. As $x$ is a point of $C-A, D+\overline{C-A}=C$; hence $D$ contains $\overline{C-C-A}$. Since $\overline{C-\overline{C-A}}$ is a sub-continuum of $A$ and contains all points of $A$ whose distance from $a$ is less than some positive $\delta$, it is identical with $A$. Thus $D$ contains $A$.

3. A Special Type of Frontier Set. Let $F=H_{1}+H_{2}$, where $H_{1}$ and $H_{2}$ are bounded continua and $F-H_{2}=H_{1}$

* See reference (C), p. 543.

$\dagger$ See reference (D), p. 155.

$\ddagger(\mathrm{F})$ C. Kuratowski, Théorie des continus irréductibles, Fundamenta Mathematicae, vol. 3, Theorem 3. 
$-H_{1} \cdot H_{2}$ and $F-H_{1}=H_{2}-H_{1} \cdot H_{2}$ are connected sets. Certain properties of this type of set and its complementary regions are easily deduced.

If $Z$ denotes the plane, it follows from the fact that $F-H_{1}$ is connected that $F-H_{1}$ lies in some one component $G_{1}$ of $Z-H_{1}$. Likewise, $F-H_{2}$ lies in one component $G_{2}$ of $Z-H_{2}$.

Now let $G^{\prime}$ be any other component of $Z-H_{1}$ and $G^{\prime \prime}$ any other component of $Z-H_{2}$. We first show that, if $G^{\prime} \cdot G_{2} \neq 0$, then $G_{2}$ contains $G^{\prime}$. For otherwise $G^{\prime}$ would contain points of $H_{2}$, the frontier of $G_{2}$, since $G^{\prime}$ is connected. This is impossible, for $G_{1}$ is the only component of $Z-H_{1}$ containing points of $H_{2}$. Likewise, either $G^{\prime \prime} \cdot G_{1}=0$ or $G_{1}$ contains $G^{\prime \prime}$.

A second property regarding these components is that either $G^{\prime} \cdot G^{\prime \prime}=0$ or else $G^{\prime}=G^{\prime \prime}$ and the frontier of $G^{\prime}$ is a part of some component of $H_{1} \cdot H_{2}$. To prove this let us assume that $G^{\prime} \cdot G^{\prime \prime} \neq 0$. Then, if $G^{\prime \prime}$ contained points not in $G^{\prime}$, it would contain points of the frontier of $G^{\prime}$, which is a part of $H_{1}$. This is impossible by the second paragraph above. Likewise, $G^{\prime}$ contains no point not in $G^{\prime \prime}$ and hence $G^{\prime}=G^{\prime \prime}$. Let $g^{\prime}$ denote the frontier of $G^{\prime}$.

Then $g^{\prime} \subseteq H_{1} H_{2}$. As $g^{\prime}$ is a continuum, it is a part of some component of $\mathrm{H}_{1} \mathrm{H}_{2}$.

With these preliminaries, we are in a position to prove the following lemma.

4. Lemma IV. Let $F$ be the union of two bounded continua $H_{1}$ and $H_{2}$ having these properties: $F-H_{1}$ and $F-H_{2}$ are connecled; ${ }^{*} H_{1} \cdot H_{2}=A+B$, where $A$ and $B$ are continua and $A \cdot B=0 ; H_{1}$ and $H_{2}$ contain sub-continua $C_{1}$ and $C_{2}$, respectively, such that $\alpha=C_{1} \cdot C_{2} \cdot A \neq 0, \beta=C_{1} \cdot C_{2} \cdot B, C_{1}$ and $C_{2}$ are irreducible between $\alpha$ and $\beta$, and $F=C_{1}+C_{2}$. Then $F$ cuts the plane and is the frontier of exactly two components of its complement.

* In view of $\S 2$, Lemma $I$, this requirement may be replaced by the hypothesis that $H_{1}$ and $H_{2}$ are irreducible about $A$ and $B$. 
Proof.* It was shown in $\$ 3$ that, if $Z$ denotes the plane, one component $G_{1}$ of $Z-H_{1}$ contains $H_{2}-(A+B)$ and one component $G_{2}$ of $Z-H_{2}$ contains $H_{1}-(A+B)$. Let $K_{1}=Z-G_{1}$ and $K_{2}=Z-G_{2}$. Then $K_{1}$ and $K_{2}$ are continua which do not cut the plane.

Now consider $K_{1} \cdot K_{2}$. As $K_{1}$ consists of $H_{1}$ and the components $G^{\prime}$ of $Z-H_{1}$ differing from $G_{1}$, and $K_{2}$ is similarly constituted, it is evident from $\$ 3$ that $K_{1} \cdot K_{2}$ consists of $A+B$ and such components $G^{\prime}$ of $Z-H_{1}$ as coincide with components $G^{\prime \prime}$ of $Z-H_{2}$. In $\S 3$ it was shown that, if $G^{\prime}=G^{\prime \prime}$, then $g^{\prime}$, the frontier of $G^{\prime}$, is a part of either $A$ or $B$. In consequence of these facts $K_{1} \cdot K_{2}$ is the sum of two continua, which may be denoted by $A^{\prime}$ and $B^{\prime}$, where $A^{\prime}$ contains $A$ and may contain one or more regions whose frontiers form a part of $A, B^{\prime}$ contains $B$ and may contain one or more regions whose frontiers form a part of $B$, and $A^{\prime} \cdot B^{\prime}=0$.

Two cases must now be considered: (a) one of the regions $G_{1}$ and $G_{2}$ is unbounded; (b) neither $G_{1}$ nor $G_{2}$ is unbounded. We shall complete the proof for the first case and then return to the second.

If $G_{1}$ is unbounded, $K_{1}$ is bounded. For otherwise a point in $K_{1}$ could be joined to one in $G_{1}$ by a broken line not cutting the bounded set $H_{1}$, which is the frontier of $G_{1}$. Likewise $K_{2}$ is bounded, if $G_{2}$ is unbounded. Thus either $K_{1}$ or $K_{2}$ is bounded. We have seen that neither of these continua separates the plane and that their divisor is two continua without common points. We can therefore apply a theorem of Miss Mullikin, $\dagger$ which shows that $K_{1}+K_{2}$ cuts the plane into exactly two regions, $R_{1}$ and $R_{2}$. Let $F_{1}$ and $F_{2}$ be the frontiers of these regions. If we can show that $F_{1}=F_{2}=F$, the theorem is proved. To this end we first observe that, since no point of $F_{1}$ or $F_{2}$ can be an inner point of $K_{1}$ or $K_{2}$, then $F_{1}$ and $F_{2}$ are parts of $H_{1}+H_{2}=F=C_{1}+C_{2}$.

* (G) This proof is a modification of the proof of Rosenthal's theorem given by S. Straszewicz in his paper Über die Zerschneidung der Ebene durch abgeschlossene Mengen, Fundamenta Mathematicae, vol. 7, p. 187.

† See reference (E), p. 160; also reference (G), §§18, 19. 
It is easily seen that $R_{1}+R_{2}=G_{1} \cdot G_{2}$. Now if $m$ is a point of $R_{1}$ and $n$ is one of $R_{2}$, there is a broken line in $G_{1}$ joining $m$ and $n$ and not cutting $H_{1}$, for $G_{1} \cdot H_{1}=0$. Thus $H_{1}$ (and in like fashion $\left.H_{2}\right)$ is not an $S(m, n)$.*

There are two sub-cases: either $F_{1}$ contains $C_{1}$ or $C_{2}$, or it does not. To fix the ideas, let $C_{1} \subset F_{1}$. Then $F_{1} \cdot C_{2}$ is not void by the previous paragraph. Since $C_{2}$ is irreducible between $\alpha$ and $\beta$, it follows from $\$ 2$, Lemma II, that, if $F_{1} \cdot C_{2} \neq C_{2}$, then $F_{1} \cdot C_{2}$ is the sum of two closed sets $M$ and $N$, such that $M \cdot N=M \cdot \beta=N \cdot \alpha=0$. (One of these, but not both, may be void.) Then $F_{1}=M+C_{1}+N$. Since $M+A \subseteq H_{2}$ and $A+C_{1} \subseteq H_{1}$, neither $M+A$ nor $A+C_{1}$ is an $S(m, n)$. Hence by a theorem of Janiszewski $M+A+C_{1}$, and consequently $M+C_{1}$, is not an $S(m, n)$. Likewise, $C_{1}+N$ is not an $S(m, n)$ and a second application of the theorem referred to gives the contradiction that $F_{1}$ is not an $S(m, n)$. Hence $F_{1} \cdot C_{2}=C_{2}$, and $F_{1}=C_{1}+C_{2}=F$.

Now suppose that neither $C_{1}$ nor $C_{2}$ is a part of $F_{1}$. Since $C_{1}$ is irreducible between $\alpha$ and $\beta, F_{1} \cdot C_{1}$ is the sum of two closed sets $M$ and $N$, such that $M \cdot N=N \cdot \alpha=M \cdot \beta=0$. Either $M$ or $N$, but not both, may be void. For the same reason $F_{1} \cdot C_{2}=P+Q$, where $P$ and $Q$ are closed and $P \cdot Q=P \cdot \beta=Q \cdot \alpha=0$. Then $F_{1}=(M+P)+(N+Q)$ and $(M+P)(N+Q)=0$, which is a contradiction, as $F_{1}$ is a continuum, unless both $M$ and $P$, or $N$ and $Q$, are void. If $M=P=0, \quad F_{1}=N+Q \subseteq N+B+Q$. As neither $N+B$ nor $B+Q$ is an $S(m, n)$, this is a contradiction.

Thus in both sub-cases $F_{1}=C_{1}+C_{2}=F$, and in like fashion $F_{2}=F$.

It now remains to take care of the situation that arises when neither $G_{1}$ nor $G_{2}$ is unbounded. If $\nu$ is a point of $G_{1}$

* A set $H$ is an $S(m, n)$ if every continuum joining $m$ and $n$ cuts $H$.

$\dagger(\mathrm{H})$ Z. Janiszewski, Sur les coupures du plan faites par des continus, Prace Matematyczno-Fizyczne, vol. 26, Theorem A: "If $P$ and $Q$ are bounded closed sets, if $P \cdot Q$ is connected, and neither $P$ nor $Q$ is an $S(m, n)$, then $P+Q$ is not an $S(m, n)$." See also Straszewicz, Fundamenta Mathematicae, vol. 4 , p. 129. 
not on $H_{2}$ and the plane is inverted with respect to $\nu$ as a center, the image $F^{*}$ of $F$ will satisfy the conditions of the first case. This follows from the facts that the correspondence between $F$ and $F^{*}$ is homeomorphic, that the image $G_{1}{ }^{*}$ of $G_{1}$ is unbounded, that the images of the frontiers of the component regions of $Z-F$ are the frontiers of the images of these regions, and that the property of irreducibility is an invariant of analysis situs. Then by the proof given above there are precisely two components, $R_{1}{ }^{*}$ and $R_{2}{ }^{*}$, of $Z-F^{*}$ which have $F^{*}$ as their frontier. Inverting again. we have the inverse images $R_{1}$ and $R_{2}$ of $R_{1}{ }^{*}$ and $R_{2}{ }^{*}$, respectively, as the only components of $Z-F$ which have $F$ as a frontier.

5. A General Theorem. It is obvious that Rosenthal's theorem is the special case of the above lemma obtained when $A$ and $B$ are points, in which case $H_{1}=C_{1}$ and $H_{2}=C_{2}$. We can, however, obtain from this lemma the following theorem, which is, aside from one exceptional case, more general than the theorem in question.

Theorem I. Let $F$ be the union of two bounded continua $C_{1}$ and $C_{2}$ having these properties: $C_{1} \cdot C_{2}=\alpha+\beta$, where $\alpha$ and $\beta$ are closed and $\alpha \cdot \beta=0$; both $C_{1}$ and $C_{2}$ are irreducible between $\alpha$ and $\beta$; and either $C_{1}$ and $C_{\varepsilon}$ are both decomposable, or $C_{1}$ is indecomposable and $C_{2}$ is decomposable and not the union of two indecomposable continua. Then $F$ cuts the plane and is the frontier of exactly two components of its complement.

Proof. This theorem is a corollary of $\$ 4$. If we let $A_{1}$ and $A_{2}$ denote the oscillatory sets of $C_{1}$ and $C_{2}$, respectively, about some point $a$ of $\alpha$, and let $B_{1}$ and $B_{2}$ have the same meaning for a point $b$ of $\beta$, it follows from $\S 2$, Lemma III, that $\alpha=A_{1} \cdot A_{2}$ and $\beta=B_{1} \cdot B_{2}$.

If both continua are decomposable, take $A=A_{2}$ and $B=B_{1}$, and set $H_{1}=A+C_{1}+B=A_{2}+C_{1}$ and $H_{2}=A+C_{2}+B$ $=C_{2}+B_{1}$. Then $H_{1} \cdot H_{2}=A+B=A_{2}+B_{1}$. If $C_{1}$ is indecomposable, take $A=A_{2}$ and $B=B_{2}$, and set $H_{1}=A+C_{1}+B=A_{2}$ $+C_{1}+B_{2}$ and $H_{2}=A+C_{2}+B=C_{2}$. Again $H_{1} \cdot H_{2}=A+B$. 
In both cases $A \cdot B=0, H_{1}$ and $H_{2}$ are irreducible about $A+B$, and $F=H_{1}+H_{2}=C_{1}+C_{2}$. Hence $\$ 4$ gives the theorem.

6. The Secondary Regions. Let $R_{1}$ and $R_{2}$ be the two principal regions, which have the frontier $F=C_{1}+C_{2}$, and let $R^{\prime}$ denote any one of the other components of $Z-F$, that is, one of the secondary regions defined by $F$ if any exist.

Since $R^{\prime}$ is a component of $Z-F$, it is a part of a component of $Z-H_{1}$ and of a component of $Z-H_{2}$. It is not a part of both $G_{1}$ and $G_{2}$, for, as seen in $\S 4, G_{1} \cdot G_{2}=R_{1}+R_{2}$. To fix the ideas let $R^{\prime} \subseteq G^{\prime}$, where $G^{\prime}$ is a component of $Z-H_{1}$ different from $G_{1}$. By definition of $G^{\prime}$ and $G_{1}, G^{\prime} \cdot H_{2}=0$; hence $\bar{G}^{\prime} \cdot F \subseteq H_{1}$. Then, if $F^{\prime}$ is the frontier of $R^{\prime}, F^{\prime} \subseteq H_{1}$. Likewise, if $R^{\prime}$ is a part of some component of $Z-H_{2}$ different from $G_{2}, F^{\prime} \subseteq H_{2}$.

Since $F^{\prime} \subset F$ and $R_{1} \cdot R^{\prime}=0$ by hypothesis, it follows that, if $m$ is a point of $R^{\prime}$ and $n$ one of $R_{1}$, then $F^{\prime}$ is an $S(m, n)$. It is also an irreducible $S(m, n){ }^{*}$ For, if $K$ is a closed sub-set of $F^{\prime}$ and $x$ is a point of $F^{\prime}-K$, then for a sufficiently small positive $\delta$ there is a circular region $U_{\delta}$ containing $x$ and points of both $R^{\prime}$ and $R_{1}$, but no point of $K$. Then $m$ and $n$ can be joined by a broken line not cutting $K$. Thus no proper closed part of $F^{\prime}$ is an $S(m, n)$.

If $F^{\prime} \subseteq H_{1}$, but $F^{\prime}$ is not a part of $C_{1}$, there are two cases to consider. If $C_{1}$ is decomposable, it follows from the definition of $H_{1}(\$ 5)$ that $F^{\prime}$ contains points of $A_{2}$ not in $C_{1}$. If $F^{\prime} \cdot\left(C_{1}-A_{1}\right) \neq 0$, we have a contradiction. For then by $\S 2$, Lemmas II and III, $F^{\prime} \supset A_{1}$. Then $F^{\prime} \cdot C_{1}$ and $F^{\prime} \cdot\left(A_{1}+A_{2}\right)$ have in common the continuum $A_{1}$. As both of these sets are proper closed parts of $F^{\prime}$, neither is an $S(m$, $n)$, which gives the contradiction that $F^{\prime}$ is not an $S(m, n)$. Thus we have $F^{\prime} \cdot\left(C_{1}-A_{1}\right)=0$ and in this case $F^{\prime} \subseteq A_{1}+A_{2}$.

If $C_{1}$ is indecomposable, then $C_{2}$ is decomposable and not the sum of two indecomposable continua. Then $A_{2} \cdot B_{2}=0$ and the argument used in the previous paragraph shows

* A set $P$ is called an irreducible $S(m, n)$ if no proper closed part of $P$ $\operatorname{sian} S(m, n)$. 
that we arrive at a contradiction if we assume that $F^{\prime}$ contains points of both $A_{2}$ and $B_{2}$ not in $C_{1}$. As $C_{1}$ is indecomposable, $A_{1}=B_{1}=C_{1}$; hence either $F^{\prime} \subseteq A_{1}+A_{2}$, or $F^{\prime} \subseteq B_{1}+B_{2}$.

The same sort of discussion is employed if $F^{\prime} \subseteq H_{2}$. Thus we have found that, if $R^{\prime}$ is a secondary region defined by the continuum $F$ of $\$ 5$, then the frontier of $R^{\prime}$ is a part either of $C_{1}$, or of $C_{2}$, or of $A_{1}+A_{2}$, or of $B_{1}+B_{2}$. For a closer determination of this frontier we need the following theorem.

7. Theorem II. Let $C$ be a bounded plane continuum irreducible between the points $a$ and $b$. Let $m$ and $n$ be two points not on $C$ and let $K$ be a closed sub-set of $C$ which is an irreducible $S(m, n)$. Then $K$ is a part or the whole of the oscillatory set of $C$ about one of its points*.

Proof. CAse I. $K$ is a continuum of condensation. Let $K_{a}$ and $K_{b}$ be the saturated semi-continua of $C-K$ containing $a$ and $b$, respectively, and let neither be void. Since $K$ is a continuum of condensation, $C=\bar{K}_{a}+\bar{K}_{b}$. Then $K \cdot \bar{K}_{a}$ and $K \cdot \bar{K}_{b}$ have a common point $c$, which is a limiting point of both $K_{a}$ and $K_{b}$. Then the reasoning in $\$ 13$ of the paper mentioned in reference (C) shows that $K$ is a part of the oscillatory set about $c$. Similar reasoning establishes the theorem for the case that either $K_{a}$ or $K_{b}$ is void.

CASE II. $K$ is not a continuum of condensation. Let us assume that $K$ contains neither $a$ nor $b$. Then there are subcontinua $A_{k}$ and $B_{k}$, irreducible between $K$ and $a$ and $b$, respectively, and $A_{k} \cdot B_{k}=0{ }^{*}$ Then $L=\overline{C-\left(A_{k}+B_{k}\right)}$ is irreducible $\ddagger$ between $A_{k}$ and $B_{k}$, and $L \subseteq K$. We first show that $L$ is indecomposable or is the union of two indecomposable continua. For, if not, there is an irreducible de-

* If every oscillatory set of $C$ is complete, the theorem can be deduced easily from Theorem 9 of a paper by R. L. Moore, Concerning upper semicontinuous collections of continua, Transactions of this Society, vol. 27 (1925), pp. 416-428.

† See reference $(\mathrm{F})$, Theorems II and IV.

$\ddagger$ See reference $(\mathrm{C}), \S 4$. 
composition* of $L$ into two proper sub-continua $M$ and $N$ such that

$$
M \cdot B_{k}=N \cdot A_{k}=0
$$

and either $M$ or $N$, say $N$, is decomposable. Obviously $N$ is irreducible between $A_{k}+M$ and $B_{k}$ and there is an irreducible decomposition of $N$ into two proper sub-continua $P$ and $Q$ such that

Then we have

$$
P \cdot B_{k}=\left(A_{k}+M\right) \cdot Q=0 .
$$

$$
\begin{aligned}
C=\left(A_{k}+M+P\right)+\left(P+Q+B_{k}\right), \\
\left(A_{k}+M+P\right) \cdot\left(P+Q+B_{k}\right)=P .
\end{aligned}
$$

Since $K \supseteq L$,

$$
K=K\left(A_{k}+M+P\right)+K\left(P+Q+B_{k}\right)
$$

is a decomposition of $K$ into two closed proper sub-sets whose common part is a continuum. This is a contradiction, by reference $(\mathrm{H})$. There are, then, two sub-cases to discuss: (a) when $L$ is the union of two indecomposable continua; (b) when $L$ is indecomposable.

(a) Let $L=M+N$, where $M$ and $N$ are indecomposable and $M \cdot B_{k}=N \cdot A_{k}=0$. Reasoning similar to the above shows that in this case $K$ contains no points not on $L$. Let $x$ be a point of $M \cdot N$; then it is not a point of $A_{k}$ or $B_{k}$. It is easily seen that $A_{k}+M$ is irreducible between $a$ and $x$, and $N+B_{k}$ between $x$ and $b$. Then, since $M$ and $N$ are indecomposable, $M$ and $N$ are the oscillatory sets of $A_{k}+M$ and $N+B_{k}$ respectively about $x$. Hence

$$
K=L=M+N
$$

is the oscillatory set of $C$ about $x$. $\dagger$

(b) Let $L$ be indecomposable. From the definition of oscillatory sets it is evident that $L$ is the oscillatory set of $C$ about any point of $C-\left(A_{k}+B_{k}\right)$; hence the theorem holds if $K=L$. If $K \neq L$, it follows by reasoning similar to

* See reference (D), p. 156.

t See reference (D), p. 153. 
that used above that either $(K-L) \cdot A_{k}$ or $(K-L) \cdot B_{k}$ is void, say the latter. Then $K \subseteq A_{k}+L$. Let $y$ be a point of $A_{k} \cdot L$; then $A_{k}$ is irreducible between $a$ and $y$, and $L+B_{k}$ is irreducible between $y$ and $b$. Let $Y^{\prime}$ be the oscillatory set of $A_{k}$ about $y$. Since $y$ does not lie on $B_{k}$, the oscillatory set of $L+B_{k}$ about $y$ is $L$. Then $Y=Y^{\prime}+L$ is the oscillatory set of $C$ about $y$.*

If $K \subseteq Y$, the theorem is proved; if not, we arrive at a contradiction. For $K$ will contain a point $z$ on $A_{k}-Y^{\prime}$. Then, if $Y_{a}^{\prime}$ is the saturated semi-continuum of $A_{k}-Y^{\prime}$ containing $a, \bar{Y}_{a}^{\prime} \supset A_{k}-Y^{\prime} \supset z . \dagger \quad$ Then $\bar{Y}_{a}^{\prime}=A_{k}$, since $A_{k}$ is irreducible between $a$ and $K$. Hence $Y^{\prime}$ is completef and $z$ is a point of $Y_{a}^{\prime}$. As $K$ joins $Y_{a}^{\prime}$ and $B_{k}$, this shows that $K \supset Y^{\prime}$. Then $K \cdot A_{k}$ and $Y^{\prime}+L$ are closed proper parts of $K$ and their divisor is the continuum $Y^{\prime}$. This is a contradiction, as their union is $K$, which is an irreducible $S(m, n)$.

Thus the proof is complete, except for the special cases where $K$ contains $a$ or $b$, or both. The above demonstration holds for these cases, if we merely replace $A_{k}$ by $a$ if $K$ contains $a$ and $B_{k}$ by $b$ if $K$ contains $b$.

8. THEOREM III. Let $F$ be the union of two bounded continua $C_{1}$ and $C_{2}$ having these properties: $C_{1} \cdot C_{2}=\alpha+\beta$, where $\alpha$ and $\beta$ are closed and $\alpha \cdot \beta=0$; both $C_{1}$ and $C_{2}$ are irreducible between $\alpha$ and $\beta$; and either $C_{1}$ and $C_{2}$ are both decomposable, or $C_{1}$ is indecomposable and $C_{2}$ is decomposable and is not the union of two indecomposable continua. Then the frontier of each secondary region determined by $F$ is a part or the whole of some oscillatory set of $C_{1}$ or $C_{2}$, or it is a part or the whole of the union of the oscillatory sets of $C_{1}$ and $C_{2}$ about some point of $\alpha$ or $\beta$.

Proof. Let $R^{\prime}$ be any secondary region and let $F^{\prime}$ be its frontier. Let $A_{1}, A_{2}, B_{1}$, and $B_{2}$ be the oscillatory sets of $C_{1}$

* See referrence (D), p. 153.

$\dagger$ See reference $(\mathrm{C}), \S 10$.

$\ddagger$ See reference $(\mathrm{C}), \S 15$. 
and $C_{2}$ about a point $a$ of $\alpha$ and a point $b$ of $\beta$, respectively.

It was shown in $\$ 6$ that, if $F^{\prime}$ is not a part of $A_{1}+A_{2}$ or $B_{1}+B_{2}$, then $F^{\prime}$ is a part of $C_{1}$ or $C_{2}$. Since $C_{1}$ and $C_{2}$ are each irreducible between $\alpha$ and $\beta$, each of them is irreducible between a point $a$ of $\alpha$ and a point $b$ of $\beta$. Hence, if $F^{\prime} \subseteq C_{1}$, the theorem of $\S 7$ shows that $F^{\prime}$ is a part of some oscillatory set of $C_{1}$. Thus the theorem is proved.

9. Conclusion. A consequence of the previous theorem is that under the hypotheses there stated the frontier of a secondary region is a part of either a continuum of condensation, an indecomposable continuum, a pair of indecomposable continua, or the union of a continuum of condensation and an indecomposable continuum. This follows at once from the proof of $\$ 7$ and the fact that the oscillatory set of a bounded irreducible continuum about one of its end points is either a continuum of condensation or an indecomposable continuum.* Obviously there are no secondary regions unless $A_{1}+A_{2}$, or $B_{1}+B_{2}$, or some oscillatory set of $C_{1}$ or $C_{2}$ cuts the plane.

The conditions imposed on the character of the continua $C_{1}$ and $C_{2}$ in $\$ 5$ take care of all possible cases with two exceptions. These are that both continua are indecomposable or that one is indecomposable and the other is the union of two indecomposable continua. With regard to the first it has been shown by Kuratowski† and Knaster $\ddagger$ that the extended theorem of Rosenthal ( $\$ 5$ ) need not hold in this case. Whether or not it holds in the second case remains to be proved.

\section{YALE UNIVERSITY}

* See reference (D), p. 155.

$\dagger$ C. Kuratowski, Sur les coupures irréductibles du plan, Fundamenta Mathematicae, vol. 6, p. 138.

$\ddagger \mathrm{B}$. Knaster, Quelques coupures singulières $d u$ plan, Fundamenta Mathematicae, vol. 7, p. 281. 